\title{
Grupos de madres durante procesos críticos en torno del nacimiento
}

Andrea Mercado *

\section{Resumen}

El artículo describe una experiencia de trabajo con grupos de madres de bebés enfermos internados en neonatología (terapia intensiva neonatal). Pretende reflexionar sobre la sesión única en el campo de la psicología de los grupos-soporte y sobre el rol del psicólogo ante situaciones críticas.

Se evaluaron cincuenta y cuatro reuniones de grupo de madres, se extrajeron quince temas recurrentes a partir de los cuales se evalúa la estrategia a seguir.

Palabras clave: Grupos de madres - Residencia - Situaciones críticas Afrontamiento - Esperanza.

\section{Abstract}

\section{«Mothers' groups during critical processes around birth»}

The article describes a clinical experience with groups of mothers of newborns who are ill and must stay in neonatology. This report tries to reflect on single session therapy within support-groups and also on psychologist's role when coping with critical situations.

Fifty four groups were studied and fifthteen recurrent themes were choosen.

Starting on them, we evaluate the strategy to follow.

Key words: Groups of mothers - residence - crisis - coping - hope.

* Licenciada en Psicología, docente en la Facultad de Psicología de la Universidad de Buenos Aires. Sargento Cabral 252 (CP. 1876) Bernal - Provincia de Buenos Aires, ARG. Tel.: 15-4997-9723. Dirección electrónica: andrea@sion.com o amercado@psi.uba.ar 
Psicodebate 3. Psicología, Cultura y Sociedad

\section{Introducción}

La siguiente experiencia forma parte del trabajo realizado por un grupo de psicólogas en el Hospital Especializado Materno Infantil “Ana Goitía” de la localidad de Avellaneda, Provincia de Buenos Aires.

La misma integra el Subprograma de Atención Psicológica al Niño Pequeño y su Familia y depende de la Facultad de Psicología de la Universidad de Buenos Aires.

El hospital atiende alrededor de 3000 partos anuales, es el tercero en importancia dentro de la Capital Federal y el Gran Buenos Aires y recibe derivaciones de otros hospitales de la provincia.

La labor realizada por el equipo es identificada dentro de esta maternidad pública con el apodo de «psicólogos de neo», ya que gran parte de la tarea se desarrolla en neonatología y sus "alrededores": sala de obstetricia, preparto, parto, recuperación, sala de internación conjunta madre-bebé, residencia de madres, terapia intensiva neonatal, unidad de cuidados intermedios, de observación. Pocas veces se entrevista a los pacientes en el consultorio ubicado entre las salas de internación.

\section{La residencia de madres}

En el primer piso del hospital, entre las salas de internación conjunta madre-bebé, existe desde el año 1992 una sala especialmente acondicionada para que las madres cuyos hijos se encuentran en terapia intensiva neonatal, terapia intermedia o unidad de observación, puedan quedarse en la institución después de obtener su alta obstétrica. Esto les permite acompañar a sus bebés y participar activamente en sus cuidados durante la internación, permitiendo a los padres el ingreso irrestricto a la sala de neonatología.

Este aspecto resulta positivo en el proceso de reparación del rol parental, afectado por la situación de internación. Se debe tener en cuenta que para estas madres la devoción corriente (Winnicott, 1991) entendida como la natural disposición competente y amorosa de las madres para cuidar al bebé no puede desplegarse, ya que estos nenonatos requieren «cuidados especiales» que necesariamente desplazan a los padres en el rol protector, llevándolos algunas veces a sentir que son la causa del problema, o culpa por el estado del bebé. (Fava Vizziello, 1993).

Las características de la institución y de la población han permitido trabajar especialmente con las madres y no con su pareja, ya que en general los padres no pueden ausentarse de sus obligaciones laborales para participar en estas actividades propuestas desde la institución.

Se intenta preservar el vínculo madre-hijo;y en ese sentido se trata de recuperar la experiencia de tener un bebé en medio del sufrimiento que supone la internación; permitiendo que las madres expresen sus angustias, temores y fantasías.

En efecto la internación de un bebé esta inmersa en la incertidumbre: por un lado los bebés son en extremo vulnerables, pero al mismo tiempo pueden mostrar una notable capacidad de recuperación, de manera que es muy complejo establecer precisiones pronósticas frente al diagnóstico inicial. 
Es sabido, tal como lo expresa Lazarus (1986) que ante una sensación mayor de incertidumbre, las personas experimentan una menor capacidad de adaptación, con la consecuente vivencia de «cansancio» que suelen expresar. Afrontar una situación requiere un esfuerzo para resolver problemas modificando o aprendiendo nuevas tareas o procedimientos, además de regular el estrés emocional: es preciso restablecer el equilibrio entre expectativas y logros posibles. (Goldson, 1979)

Tener un bebé enfermo expone a los padres a un proceso complejo que afrontarán con más o menos eficacia, en el cual se mezclan emociones ambivalentes, sentimientos de culpa, de pérdida, de fracaso, frustración, angustia. (Levobici,1983)

De acuerdo con Kaplan, cuando se trata de situaciones críticas, es favorable para el paciente recibir ayuda tan pronto como sea posible (Kaplan 1960) donde lo ideal sería la consulta dentro de la semana del evento traumático. Acompañar el proceso de internación es beneficioso en ese aspecto. En algunos casos se llega a conocer a la madre antes del parto, pero en todos los casos se realiza una entrevista individual en las primeras 24 a 72 horas del nacimiento, de acuerdo con el estado general de la puérpera. (Oiberman, 1998)

A este abordaje se le suman las reuniones en la residencia, en las que se encuentran las madres después de su alta obstétrica hasta obtener el alta del bebé. Muchas veces sucede que las madres sólo participan en una reunión, (debido al alta del bebé o a un desenlace fatal) por lo cual en cada grupo se establece una dinámica diferente: si bien la consigna inicial se relaciona con el difícil comienzo de la vida de sus bebés, se van abordando temáticas muy diversas.

La única constante en estos grupos es la relevancia del momento presente: no sólo porque el foco está puesto en la situación actual sino porque la intervención no admite esperas ni postergaciones. Las situaciones imprevistas o las emergencias en relación al nacimiento de un bebé enfermo o prematuro exigen también un esfuerzo de adaptación a los profesionales involucrados: una de la variables afectadas por este entorno es el tiempo de la sesión y la cantidad de entrevistas. En una conferencia ofrecida en Buenos Aires, el Dr. Irvin Yalom se refería a los límites que imponen las instituciones al trabajo psicoterapéutico:

«las compañías aseguradoras reducen cada vez mas los tiempos de internación, los pacientes que antes hubieran estado un mes en el hospital, ahora permanecen apenas una o dos semanas...esto implica que tal vez no tengamos oportunidad de trabajar mucho tiempo con ellos y sólo tengamos una o dos entrevistas, individuales o grupales» (Yalom, 2000).

Por otra parte el Dr. Rosenbaum (1994), trata el fenómeno de la sesión única como un hecho natural en todas las corrientes de terapias psicológicas:

«Entre el 20 y el $60 \%$ de los pacientes son atendidos en una única entrevista. (por diversos motivos del paciente o del contexto). Esto ocurre independientemente del encuadre y la orientación teórica del terapeuta. En el seguimiento muchos de estos pacientes muestran sorprendentemente buenas evoluciones. En lugar de verlos como fracasos de la psicología, se los puede considerar directamente como pacientes de 
Psicodebate 3. Psicología, Cultura y Sociedad

terapia de sesión única. En todo caso, el terapeuta tiene la responsabilidad de optimizar el efecto de la primera sesión del tratamiento, sabiendo que puede ser la última.» (1994, pág. 229 y 230.)

Considerar cuál es la intervención posible en este contexto del hospital materno infantil permitió lo siguiente:

1. tomar contacto con los grupos de madres en residencia.

2. recuperar dentro de estos grupos la idea que el saber surge no desde la imposición externa de la palabra médica sino desde la propia experiencia compartida.

3. verificar los factores terapéuticos de los grupos, (Yalom 1975) en función de los datos recopilados.

4. plantear la especificidad de las intervenciones acotadas a procesos críticos en un modelo similar al del grupo-soporte, tomando como eje los temas tratados durante las reuniones del grupo de madres en la residencia.

Afirma Stern (1978) sobre el aprendizaje del rol maternal en grupos informales: «en el grupo las madres encuentran nuevas ideas, apoyo emocional y lo que es más importante...que lo que a cada una preocupa es algo general y común que todas ellas comparten.» (pág. 203)

\section{Temas tratados en las reuniones de residencia}

Se relevaron los ejes temáticos de 54 reuniones realizadas con madres de residencia entre junio de 1998 y mayo de 2000. Las reuniones fueron coordinadas por tres terapeutas diferentes del equipo. En cada reunión se trataron entre uno y tres tópicos diferentes. De este modo se pudo distinguir 99 ejes temáticos donde se encontraron recurrencias significativas.

En una primera aproximación a estas temáticas, se logró ordenarlas de acuerdo a quince categorías centrales.

\section{Descripción de los diferentes ejes temáticos:}

\section{Neonatología:}

Las madres se lamentaban de los aspectos del servicio que percibían como una amenaza para la recuperación de los bebés o que aumentaban la sensación de incertidumbre respecto de su evolución. En detalle son:

- Desconfianza respecto de la idoneidad de los profesionales: deseo de las madres de supervisar su trabajo (2) ${ }^{1}$

1. El numero entre paréntesis representa la cantidad de veces en que fue tratado el tema 
Grupos de madres durante procesos críticos en torno del nacimiento

- Problemas dentro del servicio: temor a la propagación de infecciones (2)

- Maniobras inapropiadas de los practicantes de kinesiología (2)

- Desorganización en el manejo de la información clínica de los pacientes (1)

- Características del trato cotidiano con las enfermeras (4)

- Vivencia de maltrato (1)

Por otro lado surgen los temas en relación a la evolución del bebé internado en terapia intensiva:

- Impresión de ver a los bebés en una situación de extrema vulnerabilidad y/o deterioro y de verlos sujetos a los aparatos de neo (monitores, sondas, etc) (2)

- Sensación de incertidumbre (diagnóstica y prospectiva) (3)

- Angustia por no estar con el bebé.(1)

- Temas relacionados con la evolución clínica del bebé: engorde (1)

Estos temas están estrechamente asociados a la sensación de desplazamiento e incapacidad. En efecto, el ingreso en neonatología plantea dificultades ambientales, pero también de convivencia con los profesionales y técnicos, así como la presencia de numerosos artefactos. Muchas madres señalan la atmósfera de la neo como un espacio ajeno y aterrador, desde registros muy primarios, tales como el olor. (Con frecuencia asocian los desinfectantes con los cementerios, aunque las profesionales a cargo de la residencia no perciben lo mismo).

\section{La familia}

Dentro de esta categoría se encontró:

- Preocupación por no estar con ellos, sobre todo cuando tenían otros hijos pequeños y deciden quedarse en el hospital para cuidar de este (3)

- Celos de los hermanos (1)

- Apoyo y sostén de familiares (3)

- Conflictos de pareja (2)

- Lugar del papá (1)

- Lugar de las abuelas (1)

\section{La residencia de madres. (Convivencia)}

En este ítem se incluyen cuestiones asociadas a la residencia como lugar del hospital; las madres señalan:

- Que está lejos de neonatología: aunque se encuentra en el mismo piso (1)

- Que es bueno estar con otras mamás (2)

- Que faltan camas: a veces algunas mamás no pueden quedarse por falta de espacio (2) 
Psicodebate 3. Psicología, Cultura y Sociedad

- También hablan de las consecuencias negativas de estar mucho tiempo allí: sienten temor de «salir al exterior», como si el hospital fuese un refugio(1) y del sostén que les brinda el grupo, como compañía (1).

- También quedó registro de las veces en que la terapeuta concurrió a la sala y la encontró vacía (a pesar de ir en el horario habitual) (1), o encontró a todas las mamás durmiendo a pesar de concurrir en una hora adecuada cerca del mediodía (2)

- En una ocasión se quejaron de una mamá que molestaba (1)

\section{Vínculo Madre-Bebé}

Esta categoría incluye las reflexiones de las mamás respecto del vínculo con su propio hijo (4) pero también sus vivencias en el ejercicio del rol materno.

- Ser una buena-mala madre (estar o no con el bebé en neo) (1)

- Sintonía entre la buena evolución del bebé y el propio bienestar (2)

- Capacidad de cada mamá para cuidar del bebé en neo. Descubrimientos propios en la experiencia de interacción con el hijo (2)

- Lugar del bebé en el proyecto familiar (nombre) (1)

- Ambivalencia y rechazo: frustración respecto de los avances del tratamiento (1)

\section{Cuidados}

Hablaron sobre los particulares cuidados del bebé en neo (6), las diferencias entre el estilo del papá y la mamá (1) el temor de lastimar al bebé (1) y el cuidado del propio cuerpo inmerso en esa situación (1).

Este ítem se distingue de la dimensión vincular porque está más relacionado con el aprendizaje de nuevas capacidades para cuidar del bebé y la adaptación a la nueva situación.

\section{Esperanzasy posibilidad de proyectar}

Surge el tema de la esperanza (proyectan romances ente los bebés) (3) y la necesidad de ver los aspectos positivos dentro de la experiencia dramática (1). También reflexionan sobre el tema de ajustar las expectativas de recuperación a la realidad (2)

\section{Los médicos.}

En esta categoría se incluyen asuntos relacionados con el vínculo y la comunicación con los médicos:

- Dificultades para comprender lo que dicen (2)

- Sospechas de mala praxis (1)

- Temor ante ciertos procedimientos invasivos (2) 


\section{Muerte del bebé}

En una oportunidad, se trabaja sobre una frase dicha por una mamá: "de acá salen todos muertos". En general, se habla de sostener la presencia y acompañar el proceso del bebé. (5) Siempre aparece el tema a partir de un bebé que falleció en ese momento o el día anterior. De lo contrario el tema es eludido. En un caso llamó la atención que se evitara el tema porque había muerto un bebé en el servicio ese mismo día.

\section{Temoresfrente al alta}

Las madres se preguntan si van a poder cuidar adecuadamente al bebé fuera del contexto del hospital.(4) El temor se manifiesta sobre todo en relación con la pérdida de sostén en los casos de internaciones complejas y prolongadas.

\section{Dolor}

Si bien podría incluírse este tema dentro de otras categorías (Neo, cuidados del bebé) es interesante tomarlo por separado ya que el concepto de dolor muchas veces se deja de lado en la atención perinatal: los bebés no pueden expresar su disconformidad a la escasa implementación de métodos analgésicos, además muchas veces es tan intolerable la percepción de dolor en un recién nacido, que muchos optan defensivamente, por negarla. (3)

\section{Otras temáticas}

- Cucarachas (2) . Lo insólito de la temática llevó a ubicarla por separado.

- Lactancia (2) Llamó la atención la baja recurrencia; tal vez debido a que en muchos casos la lactancia natural debe reemplazarse por un suero o la alimentación por sonda.

- Obstetricia (2) Sólo una referencia al parto y otra a la praxis obstétrica: en general no hay tiempo en las reuniones para recordar un momento inmediato anterior: el foco es en el presente y el parto es parte del pasado.

\section{Temas aislados}

Se trató aisladamente el tema de la ansiedad, el cansancio, la culpa y la necesidad de contención (tratados en forma explícita, no como emociones asociadas).

En una oportunidad surgió un problema social de un paciente y en otro una rueda de comentarios acerca de la problemática social en general.

La temática existencial en relación a encontrarle un sentido a la experiencia de sufrimiento está registrada en una sola ocasión. 
Psicodebate 3. Psicología, Cultura y Sociedad

Tabla no1: "Temas tratados"

\begin{tabular}{ll}
\hline Temas & $\begin{array}{c}\text { Recurrencia } \\
\%\end{array}$ \\
\hline Neonatología & 19 \\
\hline Familia & 11 \\
\hline Residencia & 11 \\
\hline Vínculo & 10 \\
\hline Cuidados & 9 \\
\hline Esperanza & 6 \\
\hline Médicos & 5 \\
\hline Muerte & 5 \\
\hline Alta & 4 \\
\hline Dolor & 3 \\
\hline Insectos & 2 \\
\hline Lactancia & 2 \\
\hline Obstetricia & 2 \\
\hline Sin datos & 2 \\
\hline Otrostemas & 7 \\
\hline
\end{tabular}

\section{Gráfica I: «Recurrencia de los temas»}

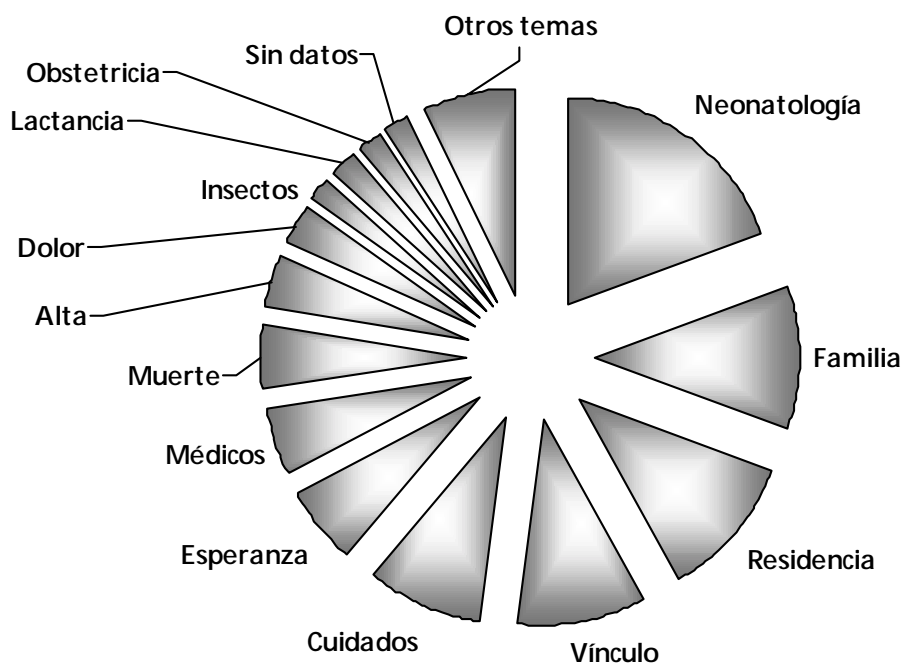




\section{Breve análisis y algunas conclusiones}

Los temas tratados permitieron extraer algunas ideas, principalmente en relación a la utilidad de esta modalidad de intervención, a los factores curativos del grupo y las características del rol del psicólogo en este marco.

Uno de los puntos más llamativos se relaciona con la circulación de información médica: en algunas ocasiones participaron médicos o enfermeras en las reuniones, pero el aspecto más relevante de la información se manifiesta cuando las madres pueden hablar sobre lo que escuchan y entienden (o no) en neonatología, y sobre las consecuencias que ellas fantasean de esos datos. A veces la incertidumbre se vuelve más insoportable que el problema en sí, y si bien la situación es de naturaleza incierta, hablar del contexto, diagnóstico y procedimientos médicos ayuda a potenciar las estrategias de afrontamiento.

Muchas veces las integrantes del equipo escucharon a alguna madre al borde de sus fuerzas diciendo que prefería que el bebé muera antes que soportar la incertidumbre en una situación prolongada de sufrimiento; extrayéndolo del contexto suena inverosímil, pero en la práctica no lo es.

Compartir las vivencias permite descentrarse del propio sufrimiento y ayudar a los demás (experiencias de universalidad y altruismo. Yalom, 1975). Esto es un efecto que se prolonga fuera de la reunión de grupo, cuando las madres tienen que enfrentar situaciones adversas, en cualquier momento del día .

La internación es vivida como una experiencia injusta y dolorosa, de manera que es importante trabajar factores existenciales como el reconocimiento de la propia precariedad o la sensación de que «a veces la vida es injusta». (Yalom, 1984)

Existe un factor en el cual se ve comprometido el rol del terapeuta: es el de infundir esperanza. Introducir esta dimensión en las reuniones con madres, constituye una herramienta más para poder atravesar la vivencia: lograr continuar psíquicamente sano a pesar del dolor. Sostenter a las madres en este proceso podría incluso prevenir conductas disfuncionales o sintomáticas que obstaculizarían el establecimiento del vínculo madrehijo. Se debe tener presente importancia de que la madre pueda ofrecer a su hijo una asistencia digna y amorosa, incluso si llegara la muerte.

Como estrategia terapéutica, la esperanza se relaciona con la confianza en los procesos y en la propia fortaleza para afrontarlos, contando con la posibilidad de esperar lo mejor. Es importante sostener a los pacientes cuando atraviesan momentos difíciles, sobre todo cuando de su resolución depende el feliz nacimiento de otra vida.

\section{Bibliografía:}

Fava Vizziello, G., Zorzi, C., Bottos, M. (1993) Los hijos de las máquinas. Buenos Aires: Nueva Visión.

Kaplan, G. (1960) Psiquiatría preventiva. Madrid 
Psicodebate 3. Psicología, Cultura y Sociedad

Lazarus, R y Folkman, S. (1986) Estrés y procesos cognitivos. Martinez Roca.

Levovici, S. (1983) El lactante, su madre y el psicoanalista. Buenos Aires: Amorrortu .

Goldson, E. (1979) Birth defects and Perinatal Death en Children Today, Julio-Agosto 1979, 8, 13-17. Colorado.

Oiberman, A. y col.(1998) Un modelo de entrevista Psicológica en situaciones críticas del nacimiento en Revista Interdisciplinaria Vol XV n ${ }^{\circ} 1-2$. Bs.As.: CIIPCA.

Paez, D.(1986) Salud mental y factores psicosociales. Madrid: Fundamentos.

Rosenmaum, R. (1994) Single session therapy en Journal of Psychotherapy Integration. (Vol.4n.3 ) 229-230.

Stern, D. (1920) La primera relación madre-hijo. Madrid: Morata. 203

Winnicott, D.(1991) Exploraciones psicoanalíticas I. Bs. As.: Paidós.

Yalom, I.(1975) The Theory and Practise of Group Psychotherapy.U.S.A.: Basic Books.

Yalom, I.(1984) Psicoterapia existencial. Barcelona: Herder.

Yalom, I.(2000) Grupos: Trama existencial y sanación . Conferencia organizada por el Centro Privado de Psicoterapias y Fundación Aiglé en la Biblioteca Nacional de Buenos Aires. 2 de mayo de 2000. 